\section{Sharper low-power STED nanoscopy by time gating}

Giuseppe Vicidomini ${ }^{1,3,4}$, Gael Moneron ${ }^{1,4}$, Kyu Y Han ${ }^{1,3,4}$, Volker Westphal ${ }^{1}$, Haisen $\mathrm{Ta}^{1}$, Matthias Reuss ${ }^{2}$, Johann Engelhardt ${ }^{2}$, Christian Eggeling ${ }^{1}$ \& Stefan W Hell ${ }^{1,2}$

Applying pulsed excitation together with time-gated detection improves the fluorescence on-off contrast in continuous-wave stimulated emission depletion (CW-STED) microscopy, thus revealing finer details in fixed and living cells using moderate light intensities. This method also enables super-resolution fluorescence correlation spectroscopy with CW-STED beams, as demonstrated by quantifying the dynamics of labeled lipid molecules in the plasma membrane of living cells.

Stimulated emission depletion (STED) $)^{1,2}$ and other fluorescence nanoscopy approaches provide relatively noninvasive insight into the nanoscale distribution of biomolecules in cells and tissues ${ }^{3}$. Nanoscopy by STED stands out by instantly providing signal from predetermined nanosized regions in the sample ${ }^{3}$, which makes it suited not only for imaging living cells but also for investigating nanoscale molecular interactions by fluorescence correlation spectroscopy (FCS $)^{4}$.

In a typical STED microscope, an excitation beam is aligned with a doughnut-shaped STED beam (Fig. 1a) to transiently turn fluorophores off ${ }^{2}$ by stimulated emission. The fluorophore remains dark if the local intensity of the STED beam exceeds $I_{\mathrm{s}}=h v /\left(\sigma \tau_{\mathrm{f}}\right)$, with $h v / \sigma$ denoting the photon energy divided by the stimulated emission cross-section ${ }^{3}$ and $\tau_{\mathrm{f}}$, the fluorescent state lifetime. Applying a doughnut crest intensity $I_{\mathrm{m}}>>I_{\mathrm{s}}$ ensures that emission is possible only in a narrow range $\Delta r \approx \lambda /\left(2 \mathrm{NA}\left(1+I_{\mathrm{m}} / I_{\mathrm{s}}\right)^{1 / 2}\right)$ around the doughnut center ${ }^{3}$, with $\lambda$ denoting the wavelength of light and NA, the numerical aperture of the lens. Scanning the beams across the sample discerns adjacent features that are as little as $\Delta r<\lambda /(2 \mathrm{NA})$ apart, because the STED beam forces them to emit sequentially.

As $\tau_{\mathrm{f}} \approx 10^{-9} \mathrm{~s}$ and $\sigma \approx 10^{-16} \mathrm{~cm}^{2}$, attaining superresolution requires $I_{\mathrm{m}}=0.1-1 \mathrm{GW} \mathrm{cm} \mathrm{cm}^{-2}$, which is conveniently supplied by continuous-wave lasers providing an average power $>300 \mathrm{~mW}^{5,6}$. To operate at lower average power, most STED nanoscopes use
Figure 1 | Principle of g-STED. (a) Microscope setup with pulsed excitation and CW-STED lasers, whose beams are combined by dichroic mirrors (gray) and which form diffraction-limited Gaussian and doughnut-shaped focal intensity distribution, respectively (inset scale bars, $200 \mathrm{~nm}$ ).

Fluorescence light (magenta) is detected by the objective lens and imaged onto a single-photoncounting (SPC) detector, whose detection events are time-gated with respect to the excitation pulses (Trigger) and registered by a computer. (b) Time-correlated single-photon counting histograms of the fluorescence of a single isolated NV center in bulk diamond for indicated CWSTED beam power $\left(P_{\text {STED }}\right)$ with experimental time sequence (top) of excitation, stimulated emission (STED) and signal detection. The time-gated detection is characterized by the time delay $T_{\mathrm{g}}$ and detection period $\Delta T$. (c) Fluorescence (arbitrary units; a.u.) detected from a single isolated NV center as a function of $P_{\text {STED }}$ for different time gates $T_{g}$ (mean \pm s.d.; $n=4$ ) and as a function of $T_{\mathrm{g}}$ for $P_{\text {STED }}=5.1 \mathrm{~mW}$ (inset, mean \pm s.d.; $n=4)$. Solid lines show theoretical fittings. (d-f) Fluorescence images of a single isolated NV center for confocal (left) and CW-STED (right, $\left.P_{\text {STED }}=47 \mathrm{~mW}\right)(\mathbf{d}), \mathrm{g}$-STED (right, $T_{\mathrm{g}}=15 \mathrm{~ns}$ ) and a fluorescence lifetime image for the CW-STED recording (left) (e) and normalized intensity profiles through the centers of the images (f). Scale bars, $200 \mathrm{~nm}(\mathbf{d}, \mathbf{e})$. Excitation, $532 \mathrm{~nm}$; repetition rate and average power $10 \mathrm{MHz}$ and $10 \mu \mathrm{W}(\mathbf{b}, \mathbf{c})$ and $20 \mathrm{MHz}$ and $11 \mu \mathrm{W}(\mathbf{d}, \mathbf{e})$, respectively. STED, $740 \mathrm{~nm}$; diffraction-limited Gaussian (b,c) and doughnut-shaped spot (d,e).

\footnotetext{
${ }^{1}$ Max Planck Institute for Biophysical Chemistry, Department of NanoBiophotonics, Göttingen, Germany. ${ }^{2}$ German Cancer Research Center, Optical Nanoscopy Division, Heidelberg, Germany. ${ }^{3}$ Present addresses: Italian Institute of Technology, Department of Nanophysics, Genoa, Italy (G.V.) and Department of Physics, University of Illinois at Urbana-Champaign, Urbana, Illinois, USA (K.Y.H.). ${ }^{4}$ These authors contributed equally to this work. Correspondence should be addressed to S.W.H. (shell@gwdg.de).
} 
Figure 2 | g-STED fluorescence nanoscopy. (a-c) Images of 40-nm-diameter yellow-green beads (a), keratin fused to the fluorescent protein citrine in a living PtK2 cell (b) and vimentin filaments in a fixed PtK2 cell labeled by immunocytochemistry with the organic dye Alexa Fluor 488 (c). Shown are CW-STED, g-STED and confocal (top right corners) recordings as well as normalized intensity (arbitrary units; a.u.) profiles along the dashed lines. Scale bars, $1 \mu \mathrm{m}$. Insets show magnified views of the marked areas, renormalized in signal intensity. Excitation, $485 \mathrm{~nm}, 80 \mathrm{MHz}$ and $11 \mu \mathrm{W}$. STED, 592 $\mathrm{nm}$ and $P_{\text {STED }}=370 \mathrm{~mW}$ (a) and $200 \mathrm{~mW}(\mathbf{b})$; gated detection: $T_{\mathrm{g}}=2 \mathrm{~ns}(\mathrm{a})$ and $1.5 \mathrm{~ns}$ (b) and $\Delta T=8 \mathrm{~ns}$.

pulsed beams for both excitation and STED $^{1,2,4,7}$. Whereas using continuouswave lasers instead is much simpler ${ }^{5}$, for a given average power, CW-STED beams yield a much lower $I_{\mathrm{m}}$ and hence a poorer resolution. Moreover, unlike in the pulsed mode in which excitation and STED can occur sequentially, in the continuous-wave mode, STED is compromised by ongoing excitation and hence a less pronounced fluorescence on-off contrast at the doughnut slope. The poorer contrast entails lower spatial frequencies in the image, masking the high spatial frequencies yielding the superresolution. It is manifested as a pedestal in the effective point spread function (E-PSF) of the microscope ${ }^{8}$, compromising the separation of object details. Here we solve this problem of CW-STED nanoscopy by implementing pulsed-laser excitation and continuous-wave laser STED in conjunction with timegated detection.

Time-gated detection has been used in fluorescence microscopy for suppressing background. Since the early days of STED nanoscopy it has been known that in pulsed STED schemes, photons should be detected after the STED pulse has left ${ }^{9}, 10$; this has been shown in a recent experiment using time-correlated single-photon counting ${ }^{11}$. Here we exploit photon arrival times to improve the spatial resolution provided by CW-STED beams so that, in conjunction with time filtering, finer details are gained with lower intensities.

In our scheme, the overlap between the excitation and the STED beam is restricted to the duration of the excitation pulse ( $<150 \mathrm{ps})$. Right afterward, only the CW-STED beam is acting and the off-switching of the fluorophore is still taking place. The longer the STED beam lasts, the more likely it becomes that a fluorophore has been turned off ${ }^{12}$. In this non-equilibrium case, the resolution $\Delta r$ not only depends on $I_{\mathrm{m}}$ but also on the time span of the STED beam action ${ }^{12}$. Another formulation is that STED reduces the lifetime of the fluorescent state from $\tau_{\mathrm{f}}=1 / k_{\mathrm{fl}}$ to $\tau=1 /\left(k_{\mathrm{fl}}+\sigma I\right)$ (Fig. 1b and Supplementary Fig. 1). Therefore, collecting photons after detection delay $T_{\mathrm{g}}>\tau$ after excitation enhances the fluorescence on-off contrast that is critical to attaining subdiffraction resolution and ensures that fluorescence light is recorded mainly from fluorophores from the doughnut center, where the STED beam is inherently weak (Fig. 1c-e).

Our gated-STED (g-STED) nanoscopy can be realized by offline processing of time-correlated single-photon counting recordings
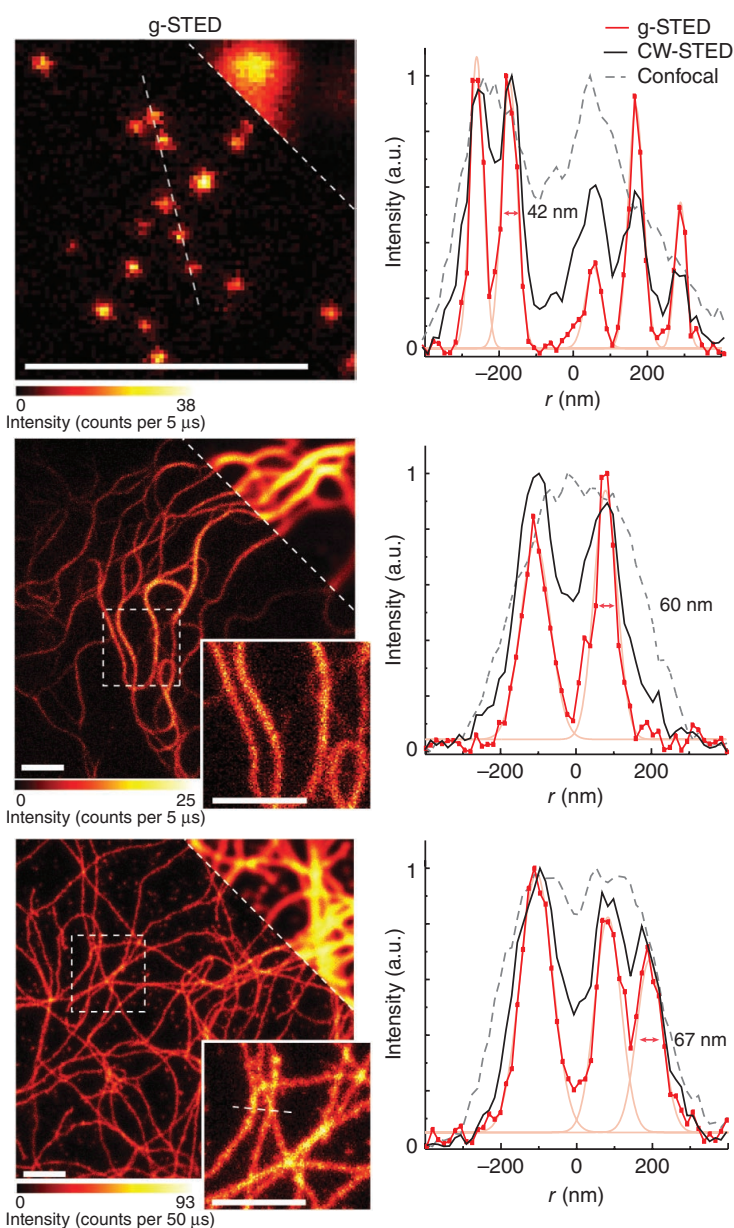

or in real time using a fast electronic gate (Supplementary Note 1). The improvement of g-STED over standard CW-STED is evidenced by the E-PSF measured with fluorescent nitrogen vacancy (NV) color centers in diamond. As $\tau$ scales inversely with the STED intensity, time-gated detection acts like a spatial filter reducing the E-PSF amplitude at the periphery and hence the E-PSF pedestal. Thus, low spatial frequency contributions from the periphery, which are large for standard CW-STED microscopy ${ }^{8}$, are suppressed (Fig. 1d-f and Supplementary Fig. 2). As the contrast between adjacent features is increased, the capability to discern features is improved (Fig. 2a and Supplementary Fig. 3). Features that are $50 \mathrm{~nm}$ apart can be clearly separated with a CW-STED beam of only $77 \mathrm{~mW}$ average power. More than twice the power is required to achieve the same separation without time-gating (Supplementary Fig. 4); yet the resulting image is more blurred.

Removing the pedestal inherently also reduces the full-width half-maximum (FWHM) (Supplementary Fig. 4). With time gating, low spatial frequencies along with their noise are discarded, which improves the resolving power without notably expanding the core bandwidth of image frequencies per se. Time gating also rejects 'desired' photons, namely those that are emitted in $T_{\mathrm{g}}$ from the doughnut center (Supplementary Fig. 4). Therefore, the increase in separation capability has to be pondered against the reduction in signal. A longer acquisition time can compensate for the concomitant decrease in signal-to-background (or signal-to-noise) ratio provided that the background noise is lower than the shot noise of the desired signal. However, both theory (Supplementary Note 1) and experiments show that the E-PSF is 

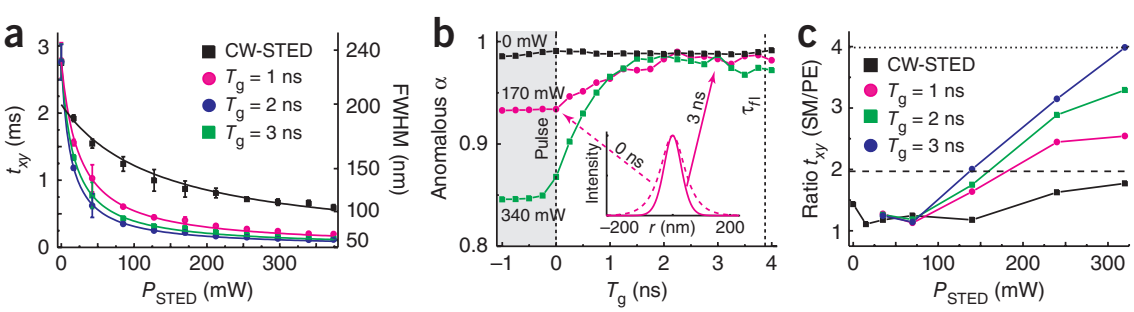

Figure 3 | g-STED-FCS. (a) Dependence of the lateral focal transit time $t_{x y}$ on $P_{\text {STED }}$ of an Atto647Nlabeled phosphoethanolamine lipid in a supported lipid bilayer, determined by FCS for indicated $T_{g}$ values (mean + s.d.; $n=6$ ). The right axis reports the FWHM of the E-PSF calculated from $\sqrt{ }\left(t_{x y}\left(P_{\text {STED }}\right) / t_{x y}(0)\right)$. Lines show theoretical fittings that match g-STED but not CW-STED nanoscopy data. (b) Dependence of the anomaly coefficient $\alpha$ of the same data on $T_{\mathrm{g}}$ for indicated $P_{\text {STED }}$ values. $\alpha \approx 1$ for Gaussian E-PSFs as for $T_{\mathrm{g}}>1 \mathrm{~ns}$ and $\alpha<1$ for Gaussian-Lorentzian shapes as for nongated CW-STED ( $T_{\mathrm{g}}<0 \mathrm{~ns}$, shaded area; dashed line, time point of maximum of excitation pulse). (c) Dependence of the ratio of the lateral focal transit time $t_{x y}(n=6$; median + s.e.m. $\approx 10 \%)$ of fluorescent lipid analogs of sphingomyelin (SM) and phosphoethanolamine (PE) in the plasma membrane of living PtK2 on $P_{\text {STED }}$ for different $T_{\mathrm{g}}$ values and CW-STED beam power. The dashed and dotted lines report the values determined from previous pulsed STED-FCS data for $\sim 70 \mathrm{~nm}$ and 40-50 nm large focal spots, respectively ${ }^{4}$. Excitation, $635 \mathrm{~nm}, 80 \mathrm{MHz}$ and $12 \mu \mathrm{W}(\mathbf{a}, \mathbf{b})$ and $8 \mu \mathrm{W}(\mathbf{c})$, STED, $770 \mathrm{~nm}$; gated detection, $\Delta T=8 \mathrm{~ns}$.

substantially sharpened with rather short $T_{\mathrm{g}}$ (for example, $5 \mathrm{~ns}$ in Supplementary Fig. 2) causing a signal reduction $<50 \%$.

To test the improvement induced by the alteration reported here, we compared CW-STED and g-STED images of a living PtK2 cell with keratin filaments tagged with the yellow fluorescent protein Citrine (Fig. 2b and Supplementary Fig. 5). We also imaged a fixed PtK2 cell whose vimentin filaments were immunolabeled with Alexa Fluor 488 (Fig. 2c and Supplementary Fig. 6). The g-STED images are clearly superior in contrast and detail. The lower CWSTED power required to obtain subdiffraction images of the same clarity (200 mW of $592 \mathrm{~nm}$ light compared to $>600 \mathrm{~mW}$ in previous recordings of similar samples ${ }^{5,6}$ ) highlights the potential of g-STED nanoscopy for live-cell imaging. We recorded similar images with several other fluorescence markers (Supplementary Fig. 6).

In combination with FCS, STED is very powerful for studying the molecular dynamics on the plasma membrane of living cells ${ }^{4,13}$. However, CW-STED-FCS has been precluded so far by the pedestal of the E-PSF defining the probing area ${ }^{8,13}$. Time gating resolves this limitation: g-STED-FCS data recordings of molecular threeand two-dimensional free diffusion (Supplementary Fig. 7 and Fig. 3a) show that the time the molecules require to cross the probing volume decreased both with increasing STED intensity $\left(I_{\mathrm{m}}\right)$ and detection delay $\left(T_{\mathrm{g}}\right)$. We obtained $\Delta r<100 \mathrm{~nm}$ and $<50 \mathrm{~nm}$ (that is, a sixfold and $>25$-fold smaller area of detection compared to the confocal one) at a CW-STED power of $60 \mathrm{~mW}$ and $350 \mathrm{~mW}$, respectively, entailing $I_{\mathrm{m}}=19 \mathrm{MW} \mathrm{cm}^{-2}$ and $109 \mathrm{MW} \mathrm{cm}^{-2}$. Removal of the pedestal is reflected by the increase of the anomaly coefficient $\alpha$ with $T_{\mathrm{g}}^{4,13}$. For free diffusion, $\alpha \approx 1$ for Gaussian E-PSFs, but without the pedestal removed it is $<1$ (Fig. $3 \mathbf{b}$ ). g-STED-FCS allowed us to use CW-STED beams to reveal the difference between the confined diffusion of sphingolipids ${ }^{4}$ and the largely free diffusion of phosphoglycerolipids in the plasma membrane of living cells (Fig. 3c and Supplementary Fig. 8). Detection of this difference is virtually impossible with $\Delta r>100 \mathrm{~nm}$, confirming our previous STED-FCS measurements using pulsed beams ${ }^{4,13}$.

As g-STED differs from reported pulsed or continuous-wave schemes by the fact that the beam-induced on-off transition is not quickly establishing equilibrium, $\Delta r$ follows an equation
(Supplementary Note 1) that has been derived for the STED concept and its generalization called reversible saturable optical fluorescence transitions (RESOLFT) with non-equilibrium transitions between the on state and the off state ${ }^{12}$. Clearly, $\Delta r$ decreases with the duration of the STED illumination before detection, which here is $T_{\mathrm{g}}$.

g-STED is applicable to virtually all fluorophores, including the fluorescent proteins, because the absolute values of $\tau_{\mathrm{fl}}$ and $T_{\mathrm{g}}$ are not critical but their ratio is. The improvement is attained without increasing the intensity $I_{\mathrm{m}}$; in fact, gating facilitates reducing $I_{\mathrm{m}}$ in practical imaging. For attaining the same separation, we applied $I_{\mathrm{m}}=100 \mathrm{MW} \mathrm{cm}^{-2}$, which is $\sim 10$ times lower than in typical pulsed systems. One also has to bear in mind that continuous-wave beams are less prone to inducing multiphoton processes known to stress the sample. In fact, of all STED modalities reported so far, g-STED provides the sharpest images with the lowest peak power. Moreover, as suitable continuous-wave (fiber) lasers are available at any visible wavelength, g-STED is destined for broad implementation.

\section{METHODS}

Methods and any associated references are available in the online version of the paper at http://www.nature.com/naturemethods/.

Note: Supplementary information is available on the Nature Methods website.

\section{ACKNOWLEDGMENTS}

We thank A. Schönle and M. Leutenegger for fruitful discussions, A. Schönle for support with the software Imspector, V. Müller and A. Honigmann for support with the FCS measurements, and U. Gemm for support with the electronics. C. Wurm, T. Gilat and E. Rothermel helped prepare samples.

\section{AUTHOR CONTRIBUTIONS}

G.V., G.M., J.E., C.E. and S.W.H. conceived and designed the study. G.V. performed theoretical studies. V.W. designed electronic components. G.V., G.M., K.Y.H., H.T. and M.R. performed experiments. G.V., G.M., K.Y.H. and C.E. analyzed data. G.V., G.M., C.E. and S.W.H. wrote the manuscript. All authors discussed the conceptual and practical implications of the method at all stages.

\section{COMPETING FINANCIAL INTERESTS}

The authors declare competing financial interests: details accompany the fulltext HTML version of the paper at http://www.nature.com/naturemethods/.

\section{Published online at http://www.nature.com/naturemethods/.}

Reprints and permissions information is available online at http://www.nature. com/reprints/index.html.

1. Hell, S.W. \& Wichmann, J. Opt. Lett. 19, 780-782 (1994).

2. Klar, T.A., Jakobs, S., Dyba, M., Egner, A. \& Hell, S.W. Proc. Natl. Acad. Sci. USA 97, 8206-8210 (2000).

3. Hell, S.W. Nat. Methods 6, 24-32 (2009).

4. Eggeling, C. et al. Nature 457, 1159-1162 (2009).

5. Willig, K.I., Harke, B., Medda, R. \& Hell, S.W. Nat. Methods 4, 915-918 (2007).

6. Moneron, G. et al. Opt. Express 18, 1302-1309 (2010).

7. Donnert, G. et al. Proc. Natl. Acad. Sci. USA 103, 11440-11445 (2006).

8. Leutenegger, M., Eggeling, C. \& Hell, S.W. Opt. Express 18, 26417-26429 (2010).

9. Schrader, M. et al. Bioimaging 3, 147-153 (1995).

10. Westphal, V. \& Hell, S.W. Phys. Rev. Lett. 94, 143903 (2005).

11. Auksorius, E. et al. Opt. Lett. 33, 113-115 (2008).

12. Hell, S.W., Jakobs, S. \& Kastrup, L. Appl. Phys., A Mater. Sci. Process. 77, 859-860 (2003).

13. Ringemann, C. et al. N. J. Phys. 11, 103054 (2009). 


\section{ONLINE METHODS}

Microscope setup. Experiments were performed on two homebuilt STED microscopes similar to those described previously $^{6,14}$. The first setup ${ }^{14}$ was used to image NV color centers and Atto647N-stained samples and to perform FCS measurements of lipid diffusion on membranes. The setup had a $532 \mathrm{~nm}$ (PicoTA, PicoQuant) or a $635 \mathrm{~nm}$ pulsed diode laser (LDH-DC-635, PicoQuant) for excitation and a titanium:sapphire laser tuned to 740-770 $\mathrm{nm}$ and operating in continuous-wave mode for STED (Mira900, Coherent). In case of continuous-wave excitation, we used a $532 \mathrm{~nm}$ continuous-wave laser (Verdi, Coherent). The fluorescence was detected with a single-photon avalanche photodiode (APD) (id100-MMF50, ID Quantique) for imaging or split by a 50:50 beam splitter and detected by two APDs (SPCMAQR-13-FC, Perkin Elmer Optoelectronics) for FCS. The detector signals were acquired by a time-correlated single-photon counting (TCSPC) board (SPC-730 or DPC-230, Becker \& Hickl) for post-acquisition, time-gating, lifetime analysis and calculation of the correlation functions. Scanning of the sample was realized using a three-axis piezo table (NanoMax, Thorlabs).

The second setup ${ }^{6}$ was used for imaging and FCS in the visible range, that is, of the yellow-green beads, Alexa Fluor 488-, Oregon Green-, Chromeo 488- or Citrine-labeled samples and Rhodamine diffusion. This microscope used a fast beam-scanning configuration with a pulsed laser module at $485 \mathrm{~nm}$ (LDH-DC-485, PicoQuant) for excitation and a continuous-wave fiber laser at $592 \mathrm{~nm}$ for STED (MPB Communications). For imaging, the fluorescence was detected with a photomultiplier (H7422PA-40, Hamamatsu) and the signal was acquired by a custom field programmable array (FPGA) board synchronized with the beam scanner ${ }^{6}$. In that case, the gated detection was performed by intercalating an additional custom-made circuit between the signal output of the detector and the input of the acquisition FPGA. In brief, the additional electronics produced a logical 'and' between the signal from the detector and a delay- and width-adjustable pulse derived from the synchronization signal from the excitation laser source. A second logical 'and' between the signal from the detector and the logical inverse of the gate was also recorded on a second channel to compare the performance of g-STED with the previously reported CW-STED (by summing the two channels). For FCS measurements, we applied an APD (id100-MMF50, Quantique) for fluorescence detection (instead of the photomultiplier) and an external correlator (Flex02-08D, http://correlator. $\mathrm{com} /$ ) in combination with the custom-build gating electronics for data acquisition (instead of the FPGA).

In both setups, the focal STED doughnut was created by placing a polymer phase plate (RPC Photonics) in the circular polarized STED beam introducing a circular phase ramp from 0 to $2 \pi$ into the back aperture of a 1.4 NA objective lens (100×, oil, Leica). Excitation and STED beams were overlapped and separated from the fluorescence by two dichroic mirrors. The fluorescence was detected by the same objective lens, cleaned up with appropriate bandpass and notch filters and collected through a multimode fiber for confocal detection.

Both for STED and excitation light we indicate the power $P$ measured at the back aperture of the objective. In the continuous-wave case, the STED intensity at the maximum of the focal spot can be estimated by $I_{\mathrm{m}}=k P_{\mathrm{STED}} / A_{\mathrm{STED}}$, where $A_{\mathrm{STED}}$ denotes the focal area and $k$ a scaling factor $k=1$ for the diffraction-limited Gaussian spot and $k=0.3$ for the doughnut-shaped spot. We determined $A_{\mathrm{STED}} \approx$ $\pi\left(\mathrm{FWHM}_{\mathrm{STED}} / 2\right)^{2}$ from the FWHM diameter, FWHM $\mathrm{STED}_{\mathrm{ST}}$, of the diffraction-limited Gaussian spot. The values of FWHM STED of $\sim 350 \mathrm{~nm}$ in the case of near-infrared STED and $\sim 270 \mathrm{~nm}$ in the case of the visible STED were measured from scattering the light on a 80-nm gold bead of subdiffraction diameter (gold colloid, En.GC80, BBinternational) in a nonconfocal mode (Fig. 1a).

NV color center and fluorescent beads. Experiments on negatively charged NV color centers ${ }^{15}$, whose absorption and emission maxima are at around $560 \mathrm{~nm}$ and $700 \mathrm{~nm}$, respectively, were performed on type IIa polycrystalline diamond grown by chemical vapor deposition $(\mathrm{CVD})^{14}$. The sample was prepared by mounting the bulk CVD diamond on a standard glass coverslip. The sample of 40-nm diameter yellow-green fluorescent particles (Yellow-green FluoSpheres, Molecular Probes; excitation and emission maxima at $505 \mathrm{~nm}$ and $515 \mathrm{~nm}$, respectively) was prepared by drop casting a 1:1,000 solution of the beads on a coverslip coated with poly-Llysine (Sigma) and mounted with Mowiol (Sigma-Aldrich).

Plasmid construction. Standard methods were used for cloning. To tag Keratin19 (Krt19) at the C terminus with Citrine ${ }^{16}$, the expression plasmid pMD-Krt19-Citrine was constructed by Gateway vector conversion (Invitrogen) from the donor vector pDONR223Krt19 (ref. 17) and the empty destination vector pMD-Citrine.

Cell culture, transfection and mounting of the cells. PtK2 cells were cultivated in DMEM medium with Glutamax and $4.5 \%$ (w/v) glucose (Invitrogen) supplemented with $50 \mu \mathrm{g} \mathrm{ml}^{-1}$ penicillin, $50 \mu \mathrm{g} \mathrm{ml}^{-1}$ streptomycin, $1 \mathrm{mM} \mathrm{Na-pyruvate} \mathrm{and} 10 \%$ (v/v) FCS (Invitrogen) at $37^{\circ} \mathrm{C}, 7 \% \mathrm{CO}_{2}$. Cells were grown overnight on glass cover slides until they reached $\sim 80 \%$ confluence. For immunofluorescence labeling, cells were fixed with ice-cold methanol $\left(-20^{\circ} \mathrm{C}\right)$ for $4 \mathrm{~min}$ followed by incubation in blocking buffer (PBS containing 1\% BSA). Then vimentin or tubulin filaments were stained using a labeling protocol involving a primary antibody (anti-vimentin mouse IgG (V9) or anti- $\beta$-tubulin mouse IgG, Sigma) and a secondary antibody (sheep anti-mouse IgG, Dianova) labeled with Alexa Fluor 488 (Invitrogen), Oregon Green (Invitrogen) or Chromeo 488 (Active Motif) (vimentin) and Atto $647 \mathrm{~N}$ (Atto-Tec). Both antibodies were diluted in blocking buffer and incubated for $1 \mathrm{~h}$ each followed by several washing steps in blocking buffer. Samples were finally embedded in Mowiol. For live-cell imaging, 1-4 d after introducing the plasmid (Nanofectin kit, PAA Laboratories), cells expressing keratinCitrine were visible and were mounted with cell culture medium without phenol red for observations on the microscope. For FCS measurements, incorporation of the fluorescent lipid analogs into the plasma membrane of living PtK2cells was accomplished via bovine serum albumin (BSA) complexes as described previously ${ }^{4}$. Briefly, cells were washed with Dulbecco's modified Eagle medium without Phenol Red buffered with 10 mM HEPES (HDMEM) and incubated with BSA-lipid complexes on ice for $30 \mathrm{~min}$, washed in cold HDMEM and incubated at $37^{\circ} \mathrm{C}$ for $4 \mathrm{~min}$ in HDMEM. Two analogs were used: Atto647N-labeled phospholipid PE (N(Atto647N)-1,2-dipalmitoyl-sn-glycero-3-phosphoethanolamine (head-group labeling), Atto-Tec) or Atto647N-labeled sphingolipid SM (N-(Atto647)-sphingomyelin (acyl chain replacement), Atto-Tec). We applied different concentrations of BSA-lipid 
complexes for incubation on ice depending on the lipid type: 5-50 nM for SM and 10-300 nM for PE.

Supported lipid layer. A single-component supported lipid bilayer was used as a control for free Brownian diffusion. We created the supported lipid bilayer on cover glass following the procedure described in reference 18 . Briefly, the lipid DOPC $(1,2-$ dioleoyl-sn-glycero-3-phosphocholine) (Avanti Polar Lipids) and the fluorescent lipid (PE) analog were mixed in organic solvents (3:1 chloroform:MeOH) at a lipid concentration of $\sim 10 \mathrm{mg} \mathrm{ml}^{-1}$. The concentration of the fluorescent lipid analogs was on the order of $10^{-4} \mathrm{~mol} \%$. Fifty microliters of the lipid stock solution were evaporated for $30 \mathrm{~min}$ under vacuum. The dry lipid film was slowly rehydrated with $50 \mu \mathrm{l}$ buffer solution $(150 \mathrm{mM} \mathrm{NaCl}$ and $10 \mathrm{mM}$ HEPES) and resuspended by vigorous vortexing. A small aliquot $(10 \mu \mathrm{l})$ of the suspension was then diluted in $140 \mu \mathrm{l}$ of the same buffer solution and sonicated for $30 \mathrm{~min}$ at $35^{\circ} \mathrm{C}$ until the buffer-lipid solution had become transparent. This solution was placed into a microscopy chamber with plasmacleaned (Femto-RF, Diener Electronic) standard microscope cover glass. A small amount of $\mathrm{MgCl}_{2}$ (to a final concentration of $3 \mathrm{mM}$ ) was added to force vesicle spreading and to initialize the bilayer formation. Thirty minutes later, the sample was thoroughly rinsed and kept under buffer solution.

FCS in solution. FCS data of three-dimensional molecular diffusion in solution were recorded for the organic dye Rhodamine 110 (Radiant Dyes) dissolved in 2,2'-thiodiethanol (TDE, SigmaAldrich; $98 \%, 2 \%$ water) to a final concentration of $100 \mathrm{nM}$. Measurements were performed at $20^{\circ} \mathrm{C}$ in $50 \mu \mathrm{l}$ samples sealed on microscope cover glass. We analyzed the normalized correlation data according to common FCS theory including diffusion and triplet-state dynamics and assuming a spatial three-dimensional Gaussian profile of the detected fluorescence ${ }^{19}$

$$
\begin{gathered}
G_{N}\left(t_{c}\right)=1+G_{N}(0)\left(\left(1+t_{c} / t_{x y}\right)^{-1}\left(1+t_{c} / t_{z}\right)^{-1 / 2}\right. \\
\left.\left(1+T /(1-T) \exp \left(-t_{c} / \tau_{T}\right)\right)\right)
\end{gathered}
$$

The lateral $t_{x y}=\mathrm{FWHM}^{2} /(8 D \ln 2)$ and axial transit time $t_{z}=$ $\mathrm{FWHM}_{z}{ }^{2} /(8 D \ln 2)$ through the three-dimensional Gaussian profile are given by the diffusion constant $D$ and the lateral and axial FWHM diameter FWHM FW $_{x y}$ and $\mathrm{FWHM}_{z}$, respectively. On-off blinking of the molecular fluorescence from the transition into the dye's dark triplet state was regarded in the FCS analysis by the characteristic parameters of an average triplet population $T$ and a triplet correlation time $\tau_{T}$.

FCS on membranes. Correlation data on membranes were recorded for diffusing fluorescent lipid analogs either in supported lipid bilayers or in the plasma membrane of living cells. Correlation data were analyzed using a model of two-dimensional anomalous subdiffusion as outlined previously $y^{4,13}$

$$
\begin{aligned}
G_{N}\left(t_{c}\right)= & 1+G_{N}(0)\left(\left(1+\left(t_{c} / t_{x y}\right)^{\alpha}\right)^{-1}\right. \\
& \left.\left(1+T /(1-T) \exp \left(-t_{c} / \tau_{T}\right)\right)\right)
\end{aligned}
$$

Here $t_{x y}$ is the focal transit time given by the diameter of the Gaussian-like assumed focal spot, the free diffusion constant and by diffusion confinements such as transient trapping. The anomalous coefficient $\alpha$ is $=1$ for free diffusion through Gaussianlike detection spots, and $<1$ for more smeared out foci such as Gaussian-Lorentzian shapes and/or for anomalous (sub)diffusion owing to confinements such as transient trapping. As for equation (1), the parameters $T$ and $\tau_{T}$ characterize transitions into the dye's dark states. The exact measurement conditions (room temperature $\left(22^{\circ} \mathrm{C}\right)$, measurements performed on the plasma membrane facing the cover glass and measurement time 10-15 s) are given in references 4,13 .

Lifetime analysis. We performed the TCSPC fluorescence lifetime analysis by means of a maximum-likelihood estimation method with Poissonian assumption for the error distribution ${ }^{20}$, which has a superior performance over least-square in analyzing low signal-to-noise ratio fluorescence decays ${ }^{21,22}$. The model fit function included a monoexponential decay and the convolution with the instrument response function. The instrument response function was measured on a scattering sample.

14. Han, K.Y. et al. Nano Lett. 9, 3323-3329 (2009).

15. Jelezko, F. \& Wrachtrup, J. Phys. Status Solidi A 203, 3207-3225 (2006).

16. Griesbeck, 0., Baird, G.S., Campbell, R.E., Zacharias, D.A. \& Tsien, R.Y. J. Biol. Chem. 276, 29188-29194 (2001).

17. Lamesch, P. et al. Genomics 89, 307-315 (2007).

18. Chiantia, S., Ries, J., Kahya, N. \& Schwille, P. ChemPhysChem 7, 2409-2418 (2006).

19. Widengren, J., Mets, U. \& Rigler, R. J. Phys. Chem. 99, 13368-13379 (1995).

20. Zander, C. et al. Appl. Phys. B 63, 517-523 (1996)

21. Maus, M. et al. Anal. Chem. 73, 2078-2086 (2001).

22. Cotlet, M. et al. J. Phys. Chem. B 105, 4999-5006 (2001). 